University of Wollongong

Research Online

Faculty of Informatics - Papers (Archive)

Faculty of Engineering and Information

Sciences

2007

\title{
A New approach to BSOFDM - Parallel Concatenated Spreading Matrices OFDM
}

Ibrahim S. Raad

University of Wollongong, ibrahim@uow.edu.au

X. Huang

University of Wollongong, huang@uow.edu.au

Follow this and additional works at: https://ro.uow.edu.au/infopapers

Part of the Physical Sciences and Mathematics Commons

\section{Recommended Citation}

Raad, Ibrahim S. and Huang, X.: A New approach to BSOFDM - Parallel Concatenated Spreading Matrices OFDM 2007.

https://ro.uow.edu.au/infopapers/3066

Research Online is the open access institutional repository for the University of Wollongong. For further information contact the UOW Library: research-pubs@uow.edu.au 


\title{
A New approach to BSOFDM - Parallel Concatenated Spreading Matrices OFDM
}

\author{
Abstract \\ This paper proposes a new approach to block spread OFDM called parallel concatenated spreading \\ matrices OFDM (PCSM-OFDM). While BSOFDM improved the overall BER performance on OFDM in \\ frequency selective channels, this new approach further improves the BER of BSOFDM by a $3 \mathrm{~dB}$ gain. \\ This is achieved through increasing the diversity of the transmitted samples by concatenating two \\ spreading matrices in parallel to BSOFDM. \\ Disciplines \\ Physical Sciences and Mathematics \\ Publication Details \\ Raad, I. \& Huang, X. (2007). A New approach to BSOFDM - Parallel Concatenated Spreading Matrices \\ OFDM. International Symposium on Communications and Information Technologies (pp. 77-81). Sydney: \\ Research Publishing Services.
}




\title{
A New Approach to BSOFDM - Parallel Concatenated Spreading Matrices OFDM
}

\author{
Ibrahim S. Raad and Xiaojing Huang \\ School of Electrical, Computer and Telecommucations Engineering \\ University of Wollongong,Wollongong NSW 2522 Australia \\ E-mail: isr01@uow.edu.au
}

\begin{abstract}
This paper proposes a new approach to Block Spread OFDM called Parallel Concatenated Spreading Matrices OFDM (PCSM-OFDM). While BSOFDM improved the overall BER performance on OFDM in frequency selective channels, this new approach further improves the BER of BSOFDM by a $3 d B$ gain. This is achieved through increasing the diversity of the transmitted samples by concatenating two spreading matrices in parallel to BSOFDM.
\end{abstract}

${ }^{1}$ Key Words-PCSM-OFDM, BSOFDM, Frequency selective channel

\section{INTRODUCTION}

Block Spread OFDM has been realized as a method to improve the BER of OFDM systems. Depending on the spreading matrix used, the performance improved and varied and in some cases showed well over 10dB gain in BER improvement [1], [6]. Primarily the Block Spread OFDM (BSOFDM) is when the full set of subcarriers are divided into smaller blocks and using spreading matrices on these blocks to spread the data so to achieve frequency diversity across frequency selective channels [2] [3] and [4]. The BSOFDM channel model is shown in Figure 1.

$$
y=C q+n
$$

The output of the receiver's FFT processor is given in Equation 1 where $y$ is the FFT output, $q \in A^{N}$ is the vector of transmitted symbols, each drawn from an alphabet $A, C$ is a diagonal matrix of complex normal fading coefficients, and $n$ is a zero mean complex normal random vector. Equalization of the received data is done through multiplication by $C^{-1}$ and then "quantized independently on each subcarrier to form the soft or hard decision $\hat{q}$ which may be further processed if the data bits are coded" [4]. There is no loss in performance when the detection is performed independently on each carrier due to the noise being independent and identically distributed with fading been diagonal [4].

The block spreading matrices are used to introduce dependence among the subcarriers. $N$ subcarriers are split into $\frac{N}{M}$ blocks, where $M=2$ is used for this example. Then each of the blocks are multiplied by a $2 \times 2$ unitary matrix $U_{2}$. The length two output vectors are interleaved using general block interleaving to ensure the symbols are statistically independent

\footnotetext{
${ }^{1}$ This research is sponsored by ARC DP 0558405
}

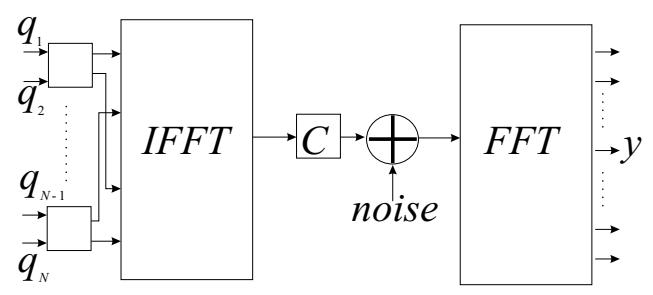

Fig. 1. Block diagram representation of the BSOFDM channel for a block length of two [4].

so as to encounter independent fading channels. This will ensure in a dispersive frequency selective channel the data is statistically less likely to become corrupted and studies and simulations have shown this to be correct.

The transmitter's IFFT has the interleaved data passed through it and this data is sent across the frequency selective channel. The data is passed through an FFT processor at the receiver and deinterleaved before using block by block processing.

The spreading matrices are generally used to increase the correlation between the transmitted symbols after the transmission has occurred. Unlike adaptive modulation schemes where depending on the system, a higher order modulation scheme is used to retransmit the data, this scheme utilizes spreading matrices to increase the correlation between the symbols, rather than retransmitting. This is depicted in Figure 2. So say at the transmission the system modulates the data using QPSK modulation, with spreading matrices a higher order modulation is used to increase correlation and therefore overall system performance.

This paper introduces a new concept for BSOFDM called Parallel Concatenated Spreading Matrices OFDM (PCSMOFDM) and simulation results have shown this new system to improve BER performance by over $3 \mathrm{~dB}$ in fading channels over classical BSOFDM or precoding OFDM. The paper has the following structure. Section II provides the system description of the new structure and provides a discussion. Section III provides simulation results comparing the PCSMOFDM with the classical approach to block spread OFDM, and finally a conclusion is given in Section IV. 


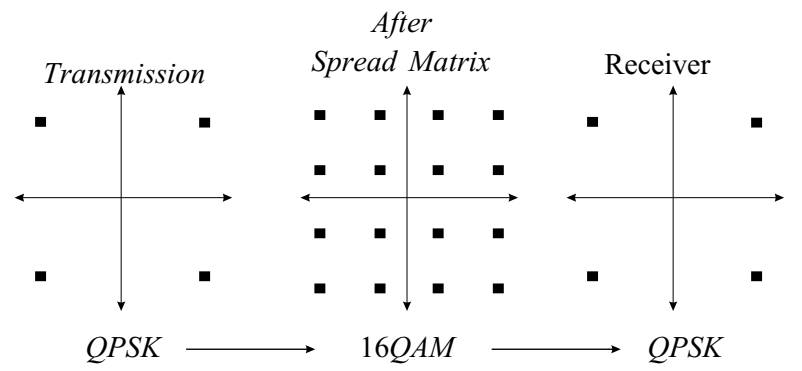

Fig. 2. The process through which the transmitted modulation is converted into a higher order modulation and then returned at the receiver.
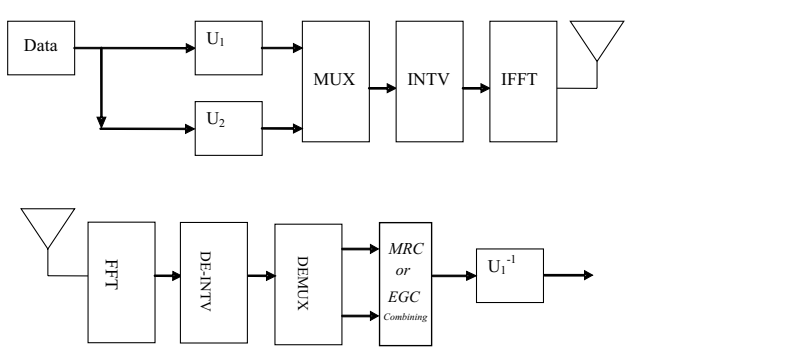

Fig. 3. The new approach to BSOFDM, Parallel Concatenated Spreading Matrices.

\section{System DESCRIPTION OF THE PCSM-OFDM}

Figure 3 depicts the block diagram of the new system described as Parallel Concatenated Spreading Matrices OFDM or PCSM-OFDM. As can be seen, and comparing this with the classical BSOFDM, the same data is split into two streams of block size $\frac{N}{M}, d_{1}$ and $d_{2}$, this is the same data and the block size $M=2$ is used for this example, and each stream is spread using a unitary spreading matrix $U$ of size $M \times M$. The streams of $M$ sized blocks can be described as $d_{1} U_{1}$. The streams are multiplexed and the same process which is applied to BSOFDM is applied to PCSM-OFDM.

At the receiver, again the same process applied to BSOFDM is applied to PCSM-OFDM except the de-multiplexing is used to separate the two streams apart. To make full use of the diversity gain the two data streams are combined before despreading takes place and the combining is done by using Maximum Ratio Combining (MRC) and can be represented by the following equation,

$$
R_{1}=\frac{\left(\alpha_{1}^{*} r_{1}+\alpha_{2}^{*} r_{2}\right)}{\left|\alpha_{1}\right|^{2}+\left|\alpha_{2}\right|^{2}}
$$

where $\alpha_{1}$ and $\alpha_{2}$ are channel weights estimated and $r_{1}$ and $r_{2}$ are the useful data from each stream. One could also apply what is known as Equal Gain Combining (EGC) where the channel weights $\alpha_{1}$ and $\alpha_{2}$ are given equal priority of 1 , and Equation 2 becomes,

$$
R_{1}=\frac{\left(r_{1}+r_{2}\right)}{2}
$$

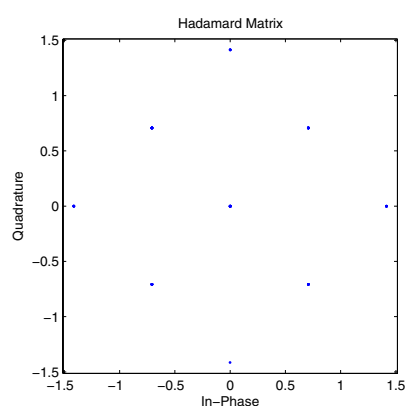

Fig. 4. After the Hadamard Matrix, the scatter plot of the data shows that it went from QPSK modulation to a higher modulation scheme.

The spreading matrices could be any matrix that the system required and the following section describes some of the spreading matrices that could be employed with this system.

\section{A. Spreading Matrices}

The spreading matrices that could be used are many, but only three is worth while mentioning. The Hadamard, Rotated Hadamard and the Rotation Spreading matrix developed in [1].

1) Hadamard Matrix: By selecting as codewords the rows of a Hadamard matrix, it is possible to produce Hadamard codes. An $N \times N$ matrix of $1^{\prime} s$ and $0^{\prime} s$ is a Hadamard matrix "such that each row differs from any other row in exactly $\frac{N}{2}$ locations. One row contains all zeros with the remainder containing $\frac{N}{2}$ zeros and $\frac{N}{2}$ ones" [5].

$\frac{N}{2}$ is the minimum distance for these codes and as an example for $N=2$, the Hadamard matrix A is

$$
U=\left[\begin{array}{cc}
1 & 1 \\
1 & -1
\end{array}\right]
$$

After the modulated data is multiplied (spread) by the Hadamard matrix, a higher order modulation scheme is created which increases the correlation between the transmitted symbols, therefore achieving a better system performance. This can be seen in Figure 4, where the modulation at the transmission was QPSK.

2) Rotated Hadamard: The rotated Hadamard codes are the same as that described above with the exception it is rotated using the rotation equation given below,

$$
U=\frac{1}{\sqrt{2}} H_{M \times M} \operatorname{diag}\left(\exp \left(\frac{j \times \pi \times m)}{C}\right)\right.
$$

where $C$ is the rotation value which the modulation rotated back on to it self, for QPSK it is four. $\mathrm{H}$ is the Hadamard matrix described above and $\mathrm{M}$ is the size of the matrix, in this example set at two. The modulation data is multiplied by the value $U$ and the rotation takes place producing a higher modulation scheme. This can be seen in Figure 5 depicting the modulated data after the rotated Hadamard matrix. The rotated Hadamard is capable of achieving 16QAM with the rotation 


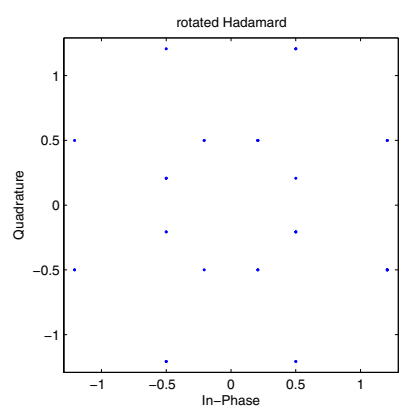

Fig. 5. After the Rotated Hadamard Matrix, the scatter plot of the data shows that it went from QPSK modulation to 16QAM.

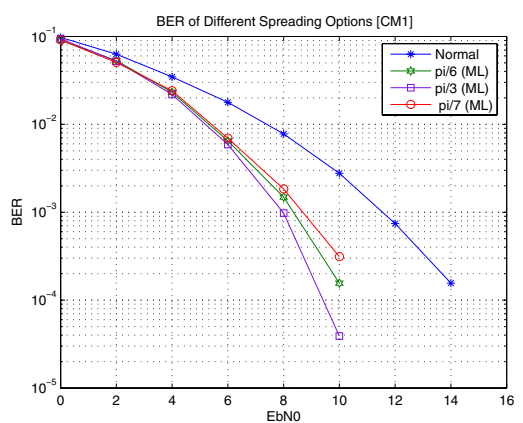

Fig. 6. Comparing the Rotation matrix with angles $\frac{p i}{6}, \frac{p i}{3}$ and $\frac{p i}{7}$ in UWB channel $C M 1[6]$.

setup in the way it is described above. So as can be seen this rotated Hadamard produces a higher order scheme than the traditional Hadamard. This is directly translated into a better BER performance in BSOFDM system of rotated Hadamard over Hadamard.

3) Rotation Spreading Matrix: The Rotation spreading matrix for BSOFDM was proposed in [1] and a number of variations and studies carried out [6], [7], [8], [9] and its structure is as follows for $U_{2}=2 \times 2$,

$$
U=\left[\begin{array}{cc}
1 & \tan (\alpha) \\
\tan (\alpha) & -1
\end{array}\right]
$$

The angle $\alpha$ is chosen depending on the system requirements and a simulation study comparing different angles performance can be seen in Figure 6 .

\section{RESUlts}

The decoder used for these simulation results is the Zero Forcing decoder and the channels used for these simulations are slow fading and frequency selective Ultra Wide Band (UWB) defined by the IEEE.802.15.3a [10]. The combining unless otherwise specified is the Equal Gain Combining (EGC) and the number of packets simulated was 10000 packets. All

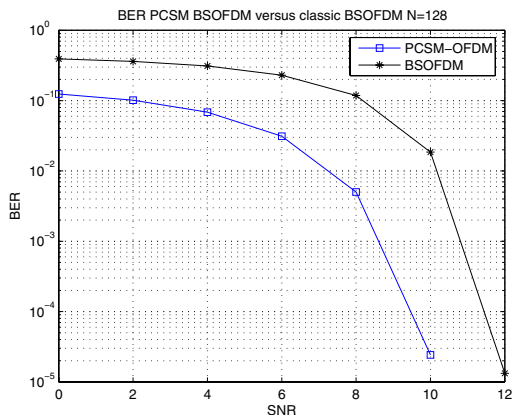

Fig. 7. PCSM-OFDM compared with BSOFDM in slow fading channel using $N=128$.

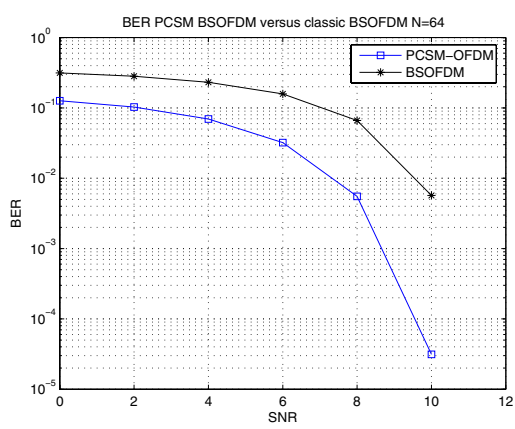

Fig. 8. PCSM-OFDM compared with BSOFDM in slow fading channel using $N=64$.

three spreading matrices discussed in Section 2 are used for these results and can be seen in Figures 7, 8,9 and 10 with the number of subcarriers $\mathrm{N}$ ranging from $N=128$ to $N=16$ across slow fading channels and as can be seen the PCSMOFDM has a greater than $3 d B$ improvement over BSOFDM in terms of BER.

Figure 9 depicts the new approach using the Rotation Spreading matrix and again it can be seen that the new structure PCSM-OFDM outperforms BSOFDM.

Figure 10 depicts the PCSM-OFDM system with the Rotated Hadamard as the spreading matrix and as can be seen, the new system outperforms the classical BSOFDM in terms of BER.

Figure 11 compares the new approach PCSM-OFDM using $N=16$ subcarriers with classical BSOFDM $N=32$ subcarriers to ensure that there is a real improvement with this new approach and not just due to an increase in samples across the channel. The $N=32$ for BSOFDM is used to compare the same sample number across the channel, and can be seen the improvement is still evident from the simulation results. It can be concluded that the improvement is not due to increase in samples. In the slow fading channel there is a greater than $4 d B$ improvement in terms of BER.

Across frequency selective channels such as the UWB 


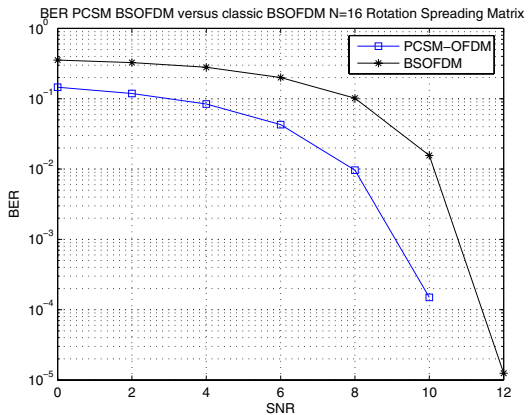

Fig. 9. PCSM-OFDM compared to BSOFDM using the Rotation Spreading matrix $\mathrm{N}=16$.

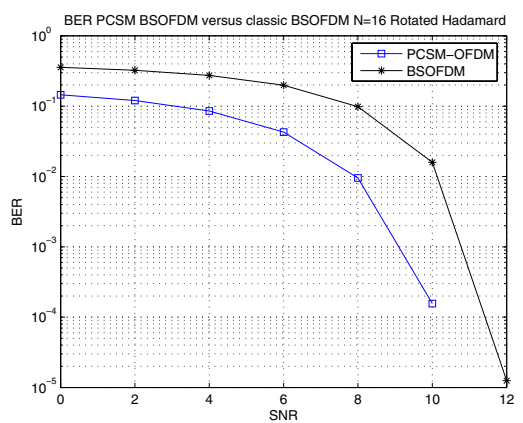

Fig. 10. PCSM-OFDM compared to BSOFDM using the Rotated Hadamard matrix $\mathrm{N}=16$.

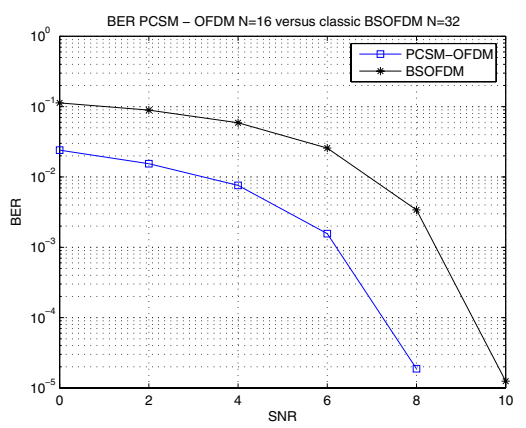

Fig. 11. PCSM-OFDM $N=16$ compared with BSOFDM $N=32$ to ensure that the correct comparison is made at the channel.

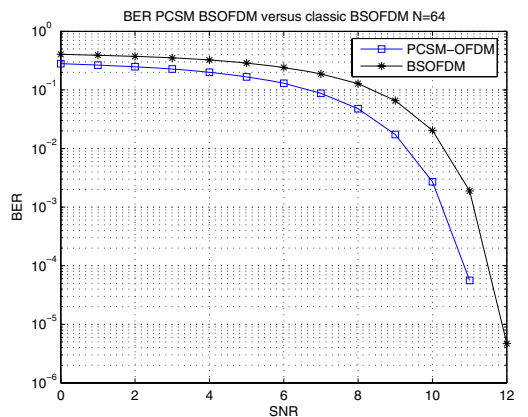

Fig. 12. PCSM-OFDM $N=64$ in UWB channel $C M 3$.

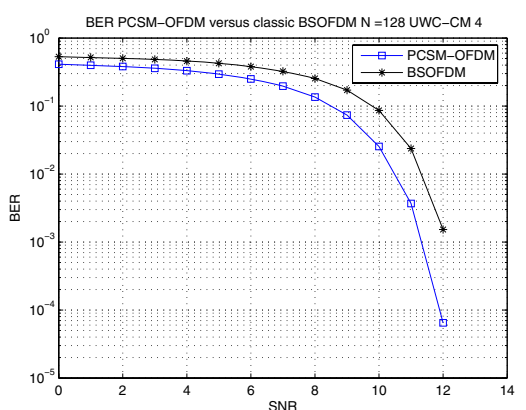

Fig. 13. PCSM-OFDM $N=128$ in UWB channel $C M 4$ compares with BSOFDM.

defined by $I E E E 802.15 .3 a$ from $C M 1$ to $C M 4$ again it can be seen in Figures 12 and 13 which depict the PCSM-OFDM across UWB channels for $N=64$ and $N=128$ subcarriers in UWB channels $C M 3$ and $C M 4$ that there is significant improvement.

In Figure 14 depicts the PCSM-OFDM when an interleaver is included and simulates if there is any improvement in UWB channel $C M 4$ with $N=128$ subcarriers. No improvement can be seen and this is due to the system being linear.

Figure 15 depicts a comparison in which there is an introduction of an interleaver and the combination been carried out before and after the de-spreading at the receiver and as can be seen no difference in terms of BER. Although, the first method in which the combination of the two streams of data before the de-spreading will ensure that the system will only use one inverse of the unitary matrix rather than two.

\section{CONCLUSION}

This paper presented a new approach to Block spread OFDM called Parallel Concatenated Spreading Matrices OFDM (PCSM-OFDM). At the transmitter the data is split into two streams of $\mathrm{M}$ sized blocks and both these streams are spread using the same unitary spreading matrix. Then the two streams are multiplexed and the normal procedure is carried out in BSOFDM and OFDM systems. At the receiver 


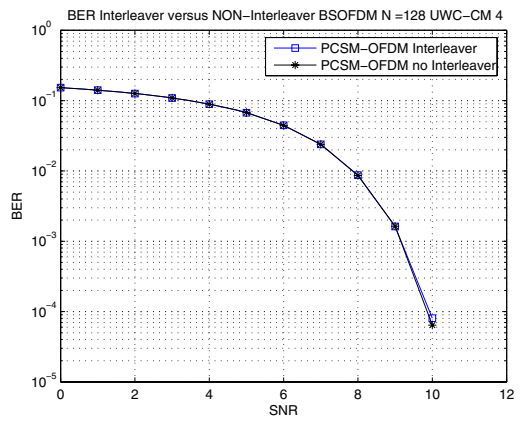

Fig. 14. PCSM-OFDM $N=128$ in UWB channel $C M 4$ compares Interleaver and non-interleaver using first combination.

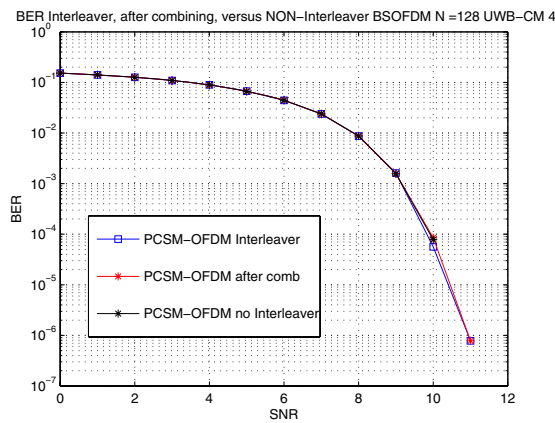

Fig. 15. PCSM-OFDM $N=128$ in UWB channel $C M 4$ compares Interleaver with combination before and after de-spreading.

after channel equalization the signal is de-multiplexed and combined using Maximum Ratio Combining (MRC) or Equal Gain Combining before the de-spreading using the inverse of the unitary matrix. In this paper Equal Gain Combining is used in the simulation results and for future work the MRC combining will be studied and discussed in which improvements are expected. From the simulation results presented, this new approach to OFDM called PCSM-OFDM outperforms the BSOFDM by greater than $4 d B$ in slow fading channels and over $3 d B$ in frequency selective channels such as UWB channels. Other combinations of PCSM-OFDM included an interleaver between the two streams at the transmitter and carrying out the de-spreading before the two streams at the receiver are combined. Both showed no improvement on the current setup, but the de-spreading after the combining would reduced complexity as you are only required to use one unitary matrix inverse. Overall, this system is recommended for wireless communication systems where OFDM and BSOFDM are used. Future study and improvement would focus on increasing the number of concatenated matrices to study if this improves the system performance and if so what would be a sufficient limit for a practical system.

\section{REFERENCES}

[1] Ibrahim Raad, Xiaojing Huang and Raad Raad, "A New Spreading Matrix for Block Spread OFDM" 10th IEEE International Conference on Communication Systems 2006 (IEEE ICCS'06), Singapore, 31 October - 3 November 2006.

[2] Ibrahim S. Raad, Xiaojing Huang, "Exploiting Time diversity to improve Block Spread OFDM", First IEEE International Conference on Wireless Broadband and Ultra Wideband Communications, AusWireless 2006, Sydney, March.

[3] Ibrahim S. Raad, Xiaojing Huang and Darryn Lowe, "Study of Spread Codes with Block Spread OFDM", First IEEE International Conference on Wireless Broadband and Ultra Wideband Communications, AusWireless 2006, Sydney, March.

[4] Michael L. McCloud, "Optimal Binary Spreading for Block OFDM on Multipath Fading Channels", WCNC / IEEE Communications Society, volume 2, 2004, 965-970, March.

[5] T.S. Rappaport, Wireless Communications - principles and practice 2002, edition second, Prentice Hall.

[6] Ibrahim Raad, Xiaojing Huang and Darryn Lowe , "A Study of different angles for the New Spread Matrix for BSOFDM in UWB channels", The Third International Conference on Wireless and Mobile Communications ICWMC 2007, March 4-9, 2007 - Guadeloupe, French Caribbean.

[7] Ibrahim Raad, Xiaojing Huang and Raad Raad, "A Study of Different Angles Higher Order Rotation Spreading Matrix for BSOFDM in UWB Channels", The second IEEE International Conference on Wireless Broadband and Ultra Wideband Communications, Aus Wireless 2007, Sydney, Australia, 27 - 30 August 2007.

[8] Ibrahim Raad, Xiaojing Huang and Raad Raad, "New Higher Order Rotation Spreading Matrix For BSOFDM", The second IEEE International Conference on Wireless Broadband and Ultra Wideband Communications, AusWireless 2007, Sydney, Australia, 27 - 30 August 2007.

[9] Ibrahim Raad, Xiaojing Huang and Darryn Lowe, "Higher Order New Matrix for Block Spread OFDM", The 14th International conference on telecommunications (ICT), 8th International conference on Communications (MICC), 14th -17th May 2007, Penang, Malaysia.

[10] Darryn W. Lowe , "Real - time FPGA Realization of an UWB transceiver physical layer" Thesis for Masters of Engineering Research at the University of Wollongong, The School of Electrical, Computer and Telecommunications Engineering, 2005. 